\title{
New photonic technologies for the treatment and diagnosis of hepatic diseases. An overview of the experimental work performed in collaboration, between Physics Institute of São Carlos and Ribeirão Preto Faculty of Medicine of the University of São Paulo ${ }^{1}$
}

\author{
Novas tecnologias fotônicas no tratamento e diagnóstico de doenças hepáticas. \\ Uma revisão dos trabalhos experimentais realizados em colaboração, entre o Instituto \\ de Física de São Carlos e a Faculdade de Medicina de Ribeirão Preto da \\ Universidade de São Paulo
}

\author{
Vanderlei Salvador Bagnato ${ }^{2}$, Cristina Kurachi ${ }^{3}, J_{u l i a n a}$ Ferreira ${ }^{4}$, Ajith Kumar Sankarankutty ${ }^{5}$, Sérgio Zucoloto $^{6}$, \\ Orlando de Castro e Silva ${ }^{7}$ \\ 1. Study performed in the Physics Institute of São Carlos (IFSC-USP), São Paulo, Brazil and Division of Gastroenterology of the Department \\ of Surgery and Anatomy of Ribeirão Preto Faculty of Medicine -University of São Paulo (FMRP-USP), Brazil. ${ }^{1}$ \\ 2. Full Professor, (IFSC-USP), São Paulo, Brazil. \\ 3. PhD, (IFSC-USP), São Paulo, Brazil. \\ 4. Master, Fellow PhD degree, (IFSC-USP), São Paulo, Brazil. \\ 5. PhD, Professor of Division of Gastroenterology of the Department of Surgery and Anatomy, (FMRP-USP), Brazil. \\ 6. Full Professor and Head of the Department of Pathology, (FMRP-USP), Brazil. \\ 7. Full Professor and Head of Division of Gastroenterology of the Department of Surgery and Anatomy, (FMRP-USP), Brazil.
}

\begin{abstract}
Recent advances in optical techniques have created a great range of possibilities for diagnosis and therapeutics in liver related diseases. With the uses of efficient light sources like lasers and LEDs (Light Emitting Diodes) it is possible to employ the light-tissue interaction to promote hepatic tissue regeneration after partial hepatectomy, to detect hepatocarcinoma and steatosis by utilizing optical fluorescence, to evaluate the metabolism of the liver during hepatic transplantation as well as to treat liver tumors. We present here an overview of the technique presently in development at the Ribeirâo Preto Faculty of Medicine - USP in cooperation with the Physics Institute of São Carlos -USP. The results obtained so far have been the subject of a list of publications and are here presented as an overview. A new perspective for modern application of optical techniques in different medical practices related to the liver is presented.
\end{abstract}

Key words: Laser. Liver Regeneration. Fluorescence. Spectroscopy. Photo Therapy.

\section{RESUMO}

Recentes avanços em técnicas ópticas têm propiciado vasto campo de possibilidades tanto para o diagnóstico quanto para a terapêutica de doenças hepáticas.Com o uso de eficientes fontes de luz como o laser e Light emitting diodes (LED) é possível utilizar a interação luz-tecido para promover a regeneração hepática após hepatectomias parciais,detectar hepatocarcinoma, esteatose e outras alterações do fígado pelo uso da fluorescência óptica,para avaliar o metabolismo hepático durante o transplante de fígado e na abordagem diagnóstica e terapêutica de alterações hepatocelulares. Os autores apresentam uma ampla revisão de técnicas atualmente em desenvolvimento na Divisão de Gastroenterologia Cirúrgica da Faculdade de Medicina de Ribeirão Preto da Universidade de São Paulo num trabalho cooperativo com o Instituto de Física de São Carlos da USP. Os resultados obtidos até agora têm sido motivo de lista de publicações que são aqui apresentados em forma de revisão. Uma nova perspectiva de moderna aplicação de técnicas ópticas em várias situações clínico-cirúrgicas relacionadas com o fígado é apresentada e amplamente discutida.

Descritores: Laser. Regeneração Hepática. Fluorescência. Espectroscopia. Fototerapia.

\section{Introduction}

Medicine is now facing a great technical revolution, where the development of fine tools for many purposes in such diverse areas as physics, chemistry and even mathematic, are finding perfect applications. In special, optics is one of those areas with a great deal of contribution. Laser and LEDs are wonderful devices that emit light with quite special properties. While lasers are coherent and preserve collimation during propagation, the light originated 
from LEDs is neither coherent nor collimated and covers a much broader range of wavelengths. Nevertheless, both lasers and LEDs are being used for different types of applications related to diagnosis and treatment of diseases or even for bio-stimulation of tissue regeneration. Conventionally, laser has been used as a cutting surgical tool in many different medical procedures. More recently, the laser has proved to be an excellent therapeutic tool, possessing biostimulatory capacity as well as being part of modern therapies such as photodynamic therapy (PDT) among others. On the other hand, LEDs can hardly be used as ablation or cutting devices, but as a source for biostimulation as well as photodynamic therapy, its finding a considerable range of applications. In special, liver has been an important target for these new techniques. Many of them are still under experimental consideration, some are already on the clinical and a few have already been incorporated into normal practice. In this article, we present an overview of some of the experiments we have performed employing new techniques involving the interaction of light with liver. We start by presenting the use of laser as a bio-stimulating device for liver regeneration. After which the photodynamic technique is introduced and examples of it application are discussed. Finally, optical biopsy is introduced and exemplified. Potentiality for further development is presented.

\section{Photo stimulation in liver regeneration}

Acting at the atomic-molecular level of cell metabolism, light has led to intriguing and exciting new observations. Low intensity visible laser light has been used to activate mitochondrial DNA replication ${ }^{1}$, to cause bioeffects on the nervous system ${ }^{2}$, to increase the regeneration of rat facial nerves $^{3}$, and to promote a stimulating effect on cell regeneration ${ }^{4}$. To verify the connection between cell activity and tissue regeneration under light application, we have performed a systematic study to investigate the effect of laser on liver regeneration. An enhancement of the mitochondrial respiration as well as the rate of mitoses in liver cells after laser radiation, without notable modification on the basal cell metabolism has been observed in a sequence of experiments performed ${ }^{5}$. The effect of low intensity visible laser radiation on the regenerative process of partially hepatectomized rats ( $70 \%$ hepatectomy) and its influence on the mitochondria has been the main target of many of our studies where the hepatocyte mitotic labeling after different intervals post partial hepatectomy reveals the variation in the mitosis rate caused by irraditation. A complementary study on the energy requirement during the liver regenerative process was also performed. In Figure 1a we show comparative results for phosphorilation, State III and State IV mitochondrial respiratory process. At 24 hours after the laser treatment a dramatic increase in ATP production per mg of mitochondrial protein is observed for the laser treated group. On the other hand, the same was not observed after longer intervals (48 and 72 hours after hepatectomy), where the laser treated and control groups show similar levels. This is an interesting result which shows a stimulating laser effect only at the earliest stages of the liver regenerating process. Similar behavior was observed for the activated and basal mitochondrial oxygen consumption, here refered to as state III and state IV, respectively (Figure $1 \mathrm{~b}$ and $1 \mathrm{c}$ ). The increase of state IV indicates an elevation of ionic flow through the inner mitochondrial membrane. And the correlation with Figure $1 \mathrm{a}$ and $1 \mathrm{~b}$ are consistent with enhancement of mitochondrial activity due to laser exposure. Laser enhanced mitochondrial activity could occur by direct light action in any of the following process: i) Increase in liver mitochondrial membrane potential; ii) Ionic flow through the internal mitochondrial membrane; iii) Activation of the heat shock protein genes; iv) Intracellular $\mathrm{pH}$; v) Alterations in amino acid transport; iv) Activation of second messengers, which signal pathways and changes in the extracellular matrix. The quantification of liver regeneration was estimated by counting the nuclei stained in the PCNA immunohystochemical assay.

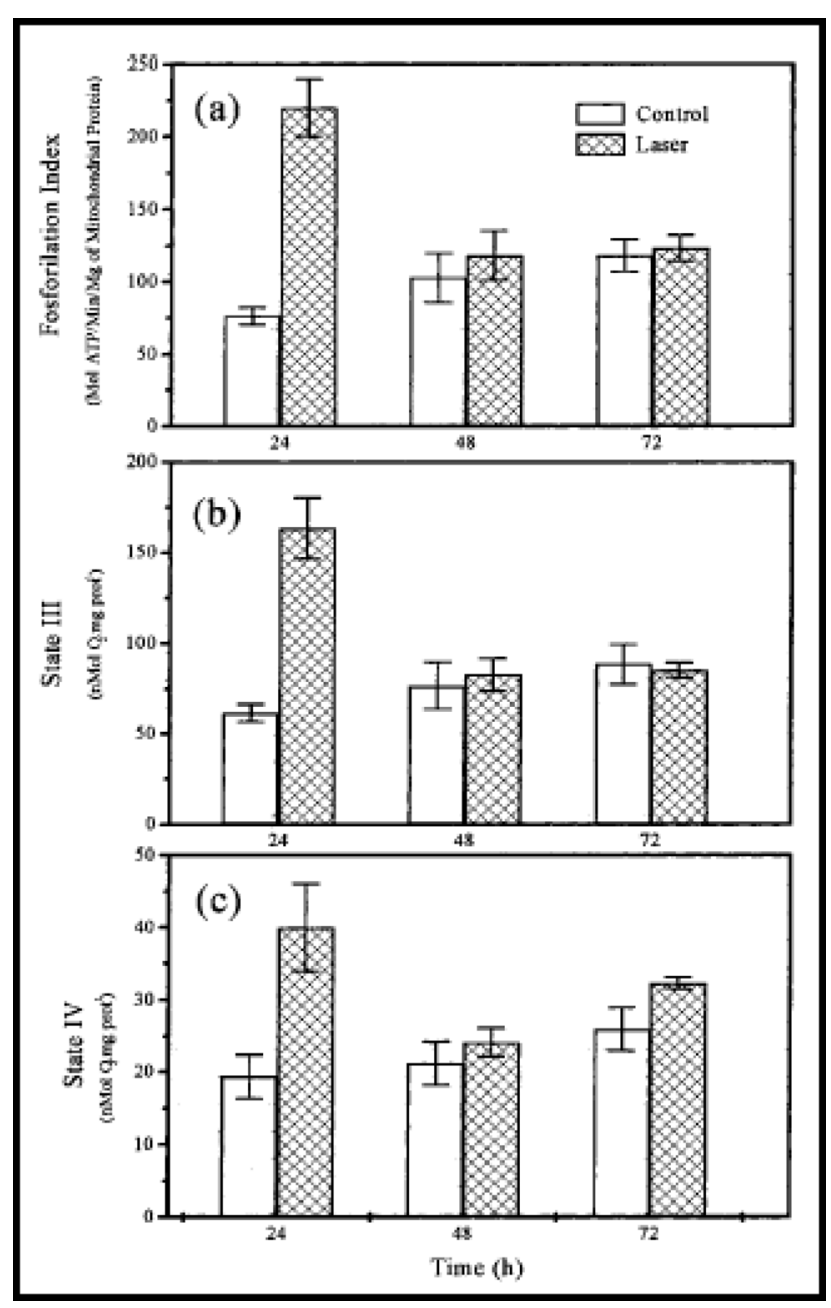

FIGURE 1 - Mitochondrial oxidative and phosphorylative activity measurement. a: Phosphorylation index. b: State III (basal mitochondrial oxygen consumption). c: State IV (mitochondrial activity stimulated with succinate). In these three measurements there is a significant increase in laser treated group in comparison with control group at 24 hours after hepatectomy. 
The increase in the labeling index of the laser treated group was remarkable after 24 hours of the procedure, showing that the laser radiation effect on hepatocyte proliferation was very noticeable at the early stages of the process. There was intense hepatocyte proliferation with vacuolization of these laser treated cells when compared to the control. The results obtained suggest that the laser irradiation promotes biological stimulatory effects on the early stages of liver regeneration without any detectable damage to the cells or it functions. That stimulus for regeneration can be of fundamental importance for improving the survival of patient after partial hepatectomy. Different ways to implement such technique for clinical application is now under consideration.

\section{Photodynamic therapy for the treatment of liver cancer}

Photodynamic Therapy (PDT) is a very powerful modality of cancer treatment with an amazing history. As pointed out in a recent article by Allison and co-workers ${ }^{6}$ in an interesting review covering more than 100 years of the use of light as a tool for therapeutics purposes in North America, PDT is the final result of many observations accumulated along decades of photo-medicine. The beauty of Photodynamic Therapy is its simplicity. Combining the photochemical properties of substances called photosensitizers with the citotoxicity of their photoproducts, we have PDT. For "ideal" photodynamic therapy a patient with a malignant tumor should develop photosensitivity caused by a photosensitizer, which must be non toxic in clinically useful doses and that is selective for the tumor, remaining in the malignant tissue more than in the normal one, promoting a high tumor to normal tissue ratio. After delivering the drug to the target, a convenient light source (most of the sources used today are lasers, but LED emitters are catching up and experiments carried out within our research program has showed the equivalence between Laser and LED for such applications) is used to illuminated the tissue, initiating the photochemical reaction that will generate extremely citotoxic oxidative species, finally resulting in death of the tissue containing the photosensitizer within the illuminated area. Here, the selectivity helps in preventing the overkill of normal cells, which is important for the recovery of the tissue. Moreover, the photodamage necessary to kill a cancer cell is lesser than that necessary to kill the normal cell. There are many mechanisms for the development of citotoxicity, the most important being the generation of singlet molecular oxygen. Singlet Oxygen are very reactive chemical species, oxidizing almost all possible biological substrate. In this manner, photosensitizers are the intermediate agents that allow the translation of light energy into the tissue destruction via chemical reaction. There are many available photosensitezers in the market as discussed in a recent article $^{7}$. They all seem to have specific clinical utility and drawbacks. The important thing is that the principles of PDT are simple and wonderful, and they work very well in practice, although, we still have not optimized the application of PDT and there still seems to be large room for improvement concerning drug and light distribution. There seems to be missing details, which are important to make the maximum of the technique. The details, are what are holding back PDT from becoming a therapeutic modality employed world wide. Many of the experimental work presently on going are trying to focus the attention on the details that will transform PDT into a more acceptable therapy. For liver PDT is being used with a lot of restriction. One of the reasons for such care is the fact that most of the photosensitizer goes to liver and selectivity between tumor cell and normal cells is not so great. We strongly believe that this limitation can be overcome. In a recent work done in our research program, the amount of light necessary to produce necrosis on the liver tissue of rats was investigated. Animals (Wistar rats) were injected with different concentrations of Photogem ${ }^{\circledR}$ (Photogem, Moscow, Russia), a hematoporphyrin derivative used as the photosensitizer. After injection their livers were illuminated with laser at different doses (Dose $=$ Intensity $\mathrm{X}$ Time) at an intensity level of $250 \mathrm{~mW} / \mathrm{cm}^{2}$ under laser light operating at $630 \mathrm{~nm}$. The incorporation of the photosensitizer in the tissue was confirmed using a fluorescence technique. According to our previous work ${ }^{8}$, where the pharmacokinetics of Photogem ${ }^{\circledast}$ was investigated in a rat model, we chose a delay time for porphyrin accumulation in the liver of about $30 \mathrm{~min}$. This delay time between injection and illumination has been adopted as standard for most of the experiments since. A medial incision in the rat abdominal region was performed to expose the liver for illumination. The right lobule was chosen for illumination, a fixed area of $1 \mathrm{~cm}^{2}$ was determined and the surrounding tissue was protected from light. The target tissue was then irradiated with the laser or the LED based device. Animals were sutured after the PDT treatment and they were maintained for $30 \mathrm{~h}$ in normal conditions after which they received anesthetized again and sacrificed. The exposed livers were removed and the macroscopic aspect of necrosis observed. Slices orthogonal to the illumination area were made for histological exams including the measurement of the depth of necrosis. The resulting depth of necrosis as a function of light dose is presented in Figure 2.

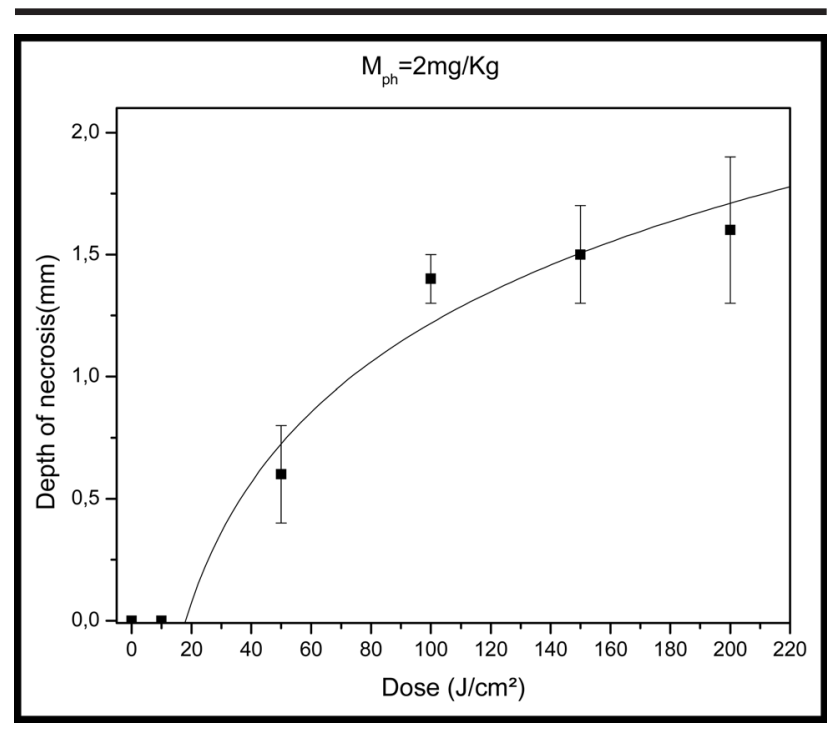

FIGURE 2 - Depth of necrosis as a function of light dose. 
For low doses, the necrosis ceases before the light. There is a threshold below which no more permanent damage is caused to the liver cells and therefore necrosis does not take place. Our investigation shows that this threshold is dependent on the drug concentration. It appears that the threshold for tumor cells is lower than that for normal cells. With this information, a way to prevent excessive damage to the organ during a cancer treatment is to use a light dose below the threshold for normal cells but above the threshold for cancer cells. With this implementation, one can apply the technique with greater security during the treatment of tumors of the liver. Experiments to demonstrate such hypothesis are presently on going to define pathways for a greater use of PDT in the treatment of liver tumors. Using such rationale, we have already performed a few cases of PDT in clinical practice, cases without alternatives in conventional practice. The results have being surprising with success well above our expectation. For the application, we used interstitial optical fiber and the illumination of the liver tumor took place from the interior towards the periphery. In this way, the health tissue is always preserved because the tumor receives the major part of light and the normal tissue below threshold. The technique of PDT for the treatment of liver cancer still needs an intense effort before it becomes the primary option for many cases. Our research group is carrying on many different detailed investigations to understand light distribution as well as drug distribution in the liver. Such information will provide the means to map out the necrotic field before the application.

Fluorescence technique for diagnosis of tumor in live regeneration

Recent technological advances have now increased the potential applications of light-tissue interactions for the recognition of diseases. One of these techniques is the fluorescence spectroscopy ${ }^{9}$, which can evaluate the physical and chemical properties of a substance by analyzing the intensity and the wavelengths of the emitted light when a well-known laser beam (in intensity and wavelength) illuminates the tissue. This technique has been of conventional use in other scientific fields for more than a hundred years, but only recently as a medical diagnosis. The development of fluorescence spectroscopy allows the detection, in real time, of alterations in tissue architecture and biochemical composition induced by tumors, tissue modifications, even at the metabolic level. The application of fluorescence technique in tissues investigation is an intensive field of research, which comprises several specialties of modern medicine ${ }^{10,11,12,13,14,15}$. The studies of fluorescence diagnostics may include a broad range of tumor types, and in this sense our work may add information to the existent literature. We have performed a study using fluorescence spectroscopy technique applied to detect hepatocarcinoma (HCC) in rats using excitation light at 442 and $532 \mathrm{~nm}$. By analyzing the ratio between the emitted fluorescence on the excitation wavelength and the broad resulting fluorescence peak we are able to identify HCC with quite high sensititvity. From our results, we believe that the fluorescence spectroscopy is a promising technique for the detection of HCC in humans in the near future. The introduction of a Figure of Merit extracted from the fluorescence spectrum is also an innovation introduced by our research team and this concept may well be a good way to identify differentiation in the spectrum. Eventually the technique could be combined with laparoscopic technique to facilitate the detection of cancer at an early stage. In the study performed, we induced hepatocarcinoma in Wistar rats using doses of 2-Acetylaminofluorene in animals submitted to a seventy percent hepatectomy. After the development of the tumor, the animals were submitted to fluorescence spectroscopy. Our fluorescence spectroscopy system is composed of: i) One spectrometer, in the range of $350 \mathrm{~nm}$ to $850 \mathrm{~nm}$; ii) One Y-shaped fiber, which delivers the laser light through one central fiber and collects the fluorescence from the tissue using six fibers distributed around the central deliver one; iii) and two excitation sources, one at $442 \mathrm{~nm}$ (He:Cd laser blue excitation) and $532 \mathrm{~nm}$ (second harmonic of Nd:YAG - green excitation). The laser for both colors is about $20 \mathrm{~mW}$, assuring no thermal effect on the incident spot. Figure 3 shows a schematic diagram of the fluorescence spectroscopy system. The typical obtained fluorescence spectrum is composed of two main features (Figure 4). A narrow peak associated with the back scattered light and a broad peak associated with all the fluorescence components. To normalize the conditions of analyses, we conventionally normalize all the spectra to the peak of back scattered light contented in the spectra. After normalization, a figure of merit is extracted from each spectrum. The figure of merit represents a characteristic of the spectra as a whole. For the present case, we defined:

$$
\begin{array}{ll} 
& \\
& F M\left(\lambda_{x}\right)=\frac{A_{1}\left(\lambda_{x}\right)}{A_{o}} \\
\text { where: } \quad & A_{0}=\int_{\lambda_{1}}^{\lambda_{2}} I(\lambda) d \lambda \\
\text { and } \quad A_{1}\left(\lambda_{x}\right)=\int_{\lambda_{3}}^{\lambda_{x}} I(\lambda) d \lambda
\end{array}
$$

where $A_{0}$ is the integration of the spectrum $(I(\lambda))$ around the signal at the excitation wavelength going from $\lambda_{1}$ to $\lambda_{2}$ (for blue excitation $\lambda_{1}=437 \mathrm{~nm}$ and $\lambda_{2}=447 \mathrm{~nm}$, for green excitation $\lambda_{1}=527 \mathrm{~nm}$ and $\lambda_{2}=540 \mathrm{~nm}$ ). $A_{1}$ is the integration of the spectrum $(\mathrm{I}(\lambda))$ around the broad fluorescence peak, going from $\lambda_{3}$ to a chosen value $\lambda_{x}$ (for blue excitation $\lambda_{3}=450 \mathrm{~nm}$, for green excitation $\lambda_{3}=545 \mathrm{~nm}$ ). Just to clarify the interpretation of $A_{0}$ and $A\left(\lambda_{x}\right)$ we show in figure 5 a typical fluorescence spectrum with all the limits of integration. For a given spectrum $\lambda_{\mathrm{x}}$ is varied, allowing identification of the best contrast between normal and HCC tissue. $A_{0}$ is the area under the first peak and $A_{1}\left(\lambda_{x}\right)$ is a variable area under the curve from $\lambda_{3}$ to $\lambda_{x}$. In figure 6 we show the value of the FM as a function of the upper limit wavelength $\left(\lambda_{\mathrm{x}}\right)$ for the excitation at $442 \mathrm{~nm}$ and $532 \mathrm{~nm}$ 
respectively. The solid squares points are for the normal liver and the open circles for the HCC. The capacity to distinguish between the normal liver tissue and the HCC through the defined FM seems remarkable. It is notable that the fluorescence of normal tissue around the tumor shows characteristics of normal liver. This suggests the possibility to using fluorescence as a technique to explore the tumor extension for surgical removal. This is now under consideration to verify the sensitivity as well as the specificity. This work has shown that fluorescence spectrum under optical excitation allows a clear distinction between normal tissue and the HCC through a figure of merit (FM), which corresponds to an appropriated integration of the spectrum (Figure 5). The obtained value for the FM is in fact a balance between the structure of the tissue and its biochemistry. This work provides more evidences to be added to the existing literature in order to push the field.

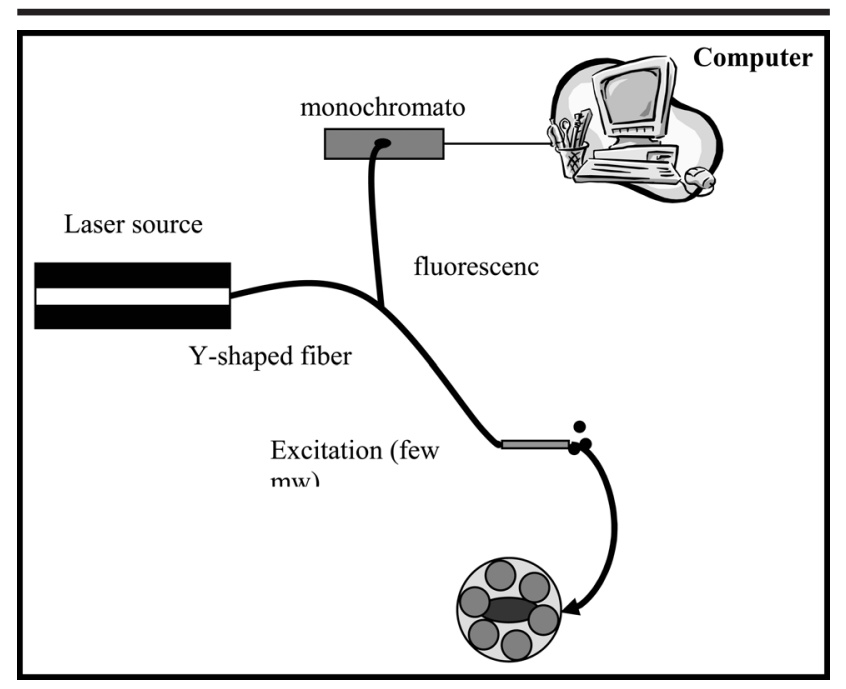

FIGURE 3 - Schematic diagram of the fluorescence spectroscopy system.

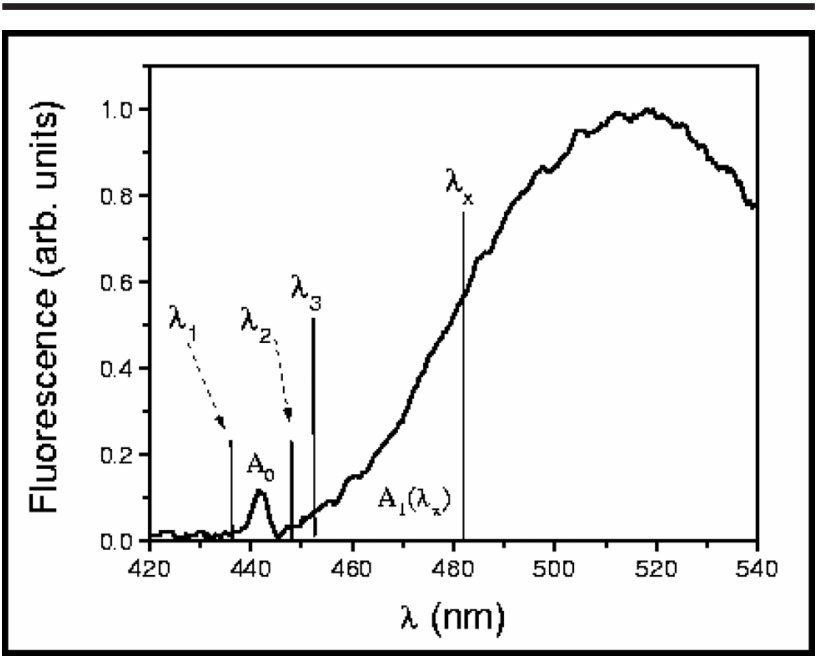

FIGURE 4 - Typical fluorescence spectrum with all the parameters involved in the calculus of FM. $\mathrm{A}_{0}$ is the area under the elastic peak and $A_{1}\left(\lambda_{x}\right)$ is the variable area under the curve from $\lambda_{3}$ to $\lambda_{\mathrm{x}}$.

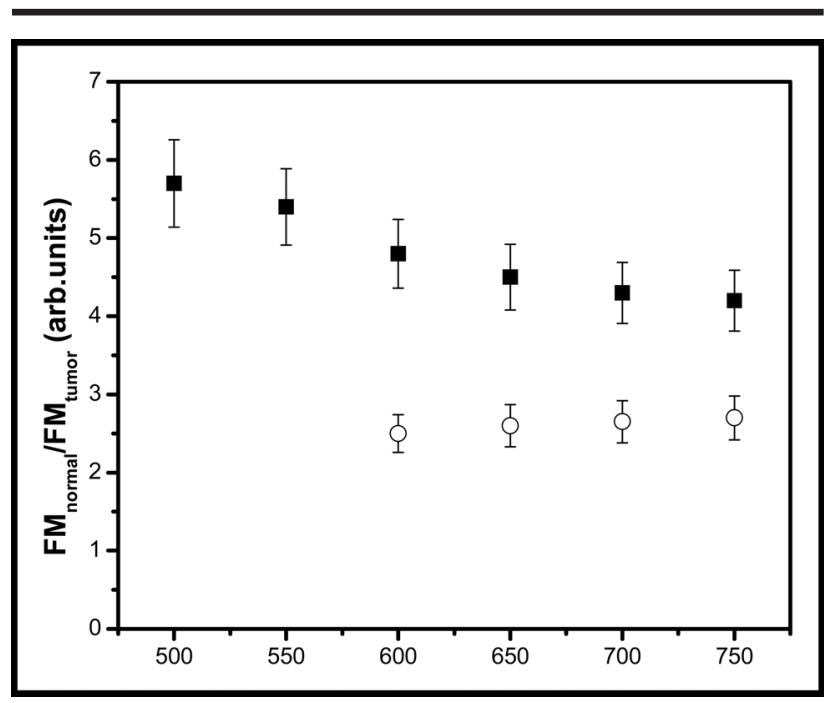

FIGURE 5 - The figure of merit as a ${ }^{\lambda(\mathrm{nm})}$ function of the upper limit wavelength $\lambda_{\mathrm{x}}$ of the integral $\mathrm{A}_{1}(\mathrm{x})$ for excitation at (a) $443 \mathrm{~nm}$ and (b) $532 \mathrm{~nm}$ respectively. The solid squares are data for the normal liver and the open circles for the hepatocarcinoma.

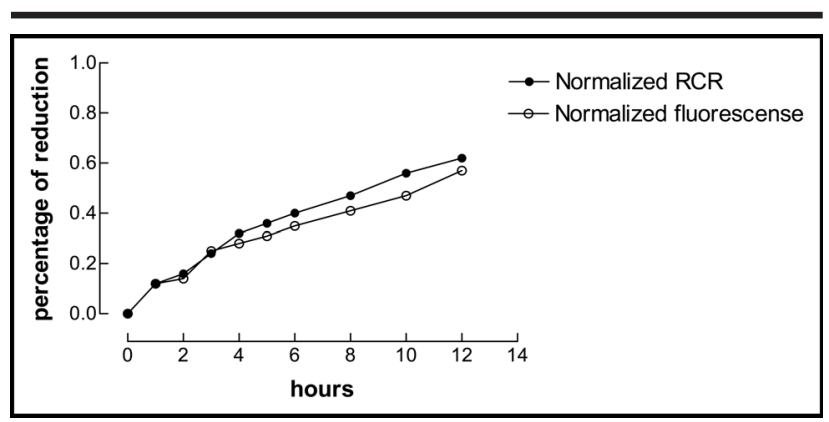

FIGURE 6 - Graph comparing the reduction of the mitochondrial respiratory control ratio (RCR) and hepatic fluorescence in the CS group, during the 12 hours after perfusion. (CS group - cold saline group)

Tests with human HCC are also on the agenda. It may, in the future, be an important coadjuvant technique in laparoscopic procedures. ${ }^{16}$

Fluorescence technique for evaluation of organs during transplantation procedure

Livers stressed in vivo, prior to storage by hypoxia, ischemia or other metabolic perturbations are called marginal livers because of doubts concerning their suitability as donor organs. The criteria for accepting or rejecting marginal livers remain largely based on the clinical experience of the individual surgeon. Quantitative and rapid techniques to help with this decision are still to be developed. Optical Spectroscopy has the potential to provide important diagnostic information about tissue conditions. Light signals can be delivered and collected via optical fibers showing aspects not commonly found in other techniques. Information can thus be gathered in situ instead of bringing the tissue to the instrument, part of the instrument can be 
brought to the tissue. Further, data can be collected and analyzed in a fraction of a second, allowing the possibility of real-time feedback. In addition, spectroscopic signals can indicate biochemical changes, and these generally precede the morphological changes observed in histology. But perhaps most important of all, the information provided by spectroscopy is quantitative, and thus it can bring an added degree of objectivity to the process of diagnosing disease. Various spectroscopic techniques can be used to diagnose tissue. Reflectance, fluorescence, infrared absorption, Raman spectroscopy and others have been employed. Each of these has its own special features and potential applications. ${ }^{17}$ Laser-induced Fluorescence (LIF) has been shown to be a very sensitive analytical technique for biochemical analysis at ultra trace levels. This technique has also been demonstrated to be a useful tool for monitoring DNA damage and for cancer diagnostics. ${ }^{18,19,20}$ Recently LIF has been used experimentally to analyze the viability of porcine hearts after perfusion with storage solutions. ${ }^{21}$ We have conducted an experiment to analyze, experimentally, the applicability of LIF in evaluating the viability of rat liver once perfused with saline solution. Mitochondrial function and cellular ATP content of the liver were used as indicators of it's metabolic status. The spectroscopic findings were then compared on a time scale with the mitochondrial function. Therefore, the aim of the study was to verify LIF's capacity to detect changes taking place in the organ. Structural changes usually occur at later stages and frequently denote irreversible damage. Laser Induced Fluorescence (LIF) was measured before and soon after perfusion with the storage solution. Thereafter, the measurements were taken hourly for a period of 12 hours. For each measurement, LIF was obtained from three different points of three lobes and the average of these nine points was used to plot the graph. Like the LIF measurements, mitochondrial function and cellular ATP content of the liver were evaluated before and soon after perfusion, then hourly for 12 hours. Each measurement of mitochondrial function was obtained from rats so that the average could be plotted. Compared on a time scale, initially there seems to be a reduction in the cellular ATP content, followed by progressive mitochondrial swelling, diminished RCR and finally by the loss of mitochondrial membrane potential. For the mitochondrial function as well as the ATP content, time zero was considered as the moment soon after perfusion. This coincides with the knowledge that the differences in temperature of the perfusion solution can cause a difference in the degree of damage to the organ. The typical fluorescence spectrum has a common peak centered at the excitation wavelength followed by a broad peak at longer wavelengths, which extends from just above $532 \mathrm{~nm}$ to around $800 \mathrm{~nm}$. This broad peak of fluorescence has various contributions. We believe that many endogenous chromophors contribute to this peak. The fluorescence of reduced nicotinamide adenine dinucleotide (NADH) may have special contribution as it is an essential part of the cellular energy metabolism, and it could therefore reveal the energy status of the cell. Although isolated NADH does not show prominent fluorescence under green $(532 \mathrm{~nm})$ excitation, in the intact organ (hepatocellular medium) NADH fluorescence may behave in a significantly altered manner, to the point of responding to green excitation. This, however, remains to be investigated. In order to obtain better organ preservation and consequently increase the donor pool, there is a lot still to be learned about the metabolism after organ perfusion and removal as well as about how individual chromophors in a cellular environment contribute to the fluorescence spectrum. To assure comparability in equivalent conditions of light and tissue coupling, we normalized all spectra at the excitation wavelength (reducing the background scattering). In other words, in each spectrum, the excitation peak received the value 1 . After this normalization procedure, we notice that the overall intensity of fluorescence decreased with time. That indicates a possible variation in biochemical activity and/or a possible modification of the cellular structure. To quantify the fluorescence variation, we calculated the area of the broad fluorescence spectra and calculated its reduction as time passed. As usual in the processing of fluorescence spectrum, we defined:

$$
A_{t}=\int_{\lambda_{1}}^{\lambda_{2}} I(\lambda) d \lambda
$$

where $A_{t}$ is the integration of the spectrum $(I(\lambda))$ from $\lambda_{1}=550 \mathrm{~nm}$ to $\lambda_{2}=800 \mathrm{~nm}$, for the observation taken place at a time $t$ after perfusion. The percentage of fluorescence reduction (PFR) observed in the tissue as the time pass by can be obtained calculating

$$
\mathrm{PFR}=100(\mathrm{At}-\mathrm{A} 0) / \mathrm{A}_{0}
$$

The RCR has reduced to about $60 \%$ if it's original value in 12 hours. This does not necessarily mean that the organ has undergone permanent irreversible damage. At present, we do not know at what RCR level the damage is still reversible. That will be the subject of future studies. The focus of comparison with the fluorescence spectrum was the RCR as it showed the greatest similarity with the changes in spectral analysis on a time scale. Considering that fluorescence is related to tissue activity, the PFR can be compared to the RCR. The behavior is very similar to the $\mathrm{RCR}$, indicating that there is a real correlation between the time variation of RCR and that of fluorescence. RCR represents only the mitochondrial activity, whereas the fluorescence has innumerous contributions, i.e. as long as there are molecules fluorescence will always exist. Therefore a numerical constant must correlate both quantities and this can be obtained in the following manner:

$$
(\mathrm{RCR})_{\mathrm{F}}=\mathrm{g} \text { PFR }
$$

where the subscript $F$ represents the value of $R C R$ obtained by fluorescence. In our case the numerical constant $g=1.3$. The figure 6 compares the $(\mathrm{RCR})_{\mathrm{F}}$ with the RCR obtained from the respiratory assay in the CS group. The similarity between the values is very remarkable proving the viability of using fluorescence spectra as a tool for following changes in liver grafts. We have recently performed the first clinical case of induced fluorescence to follow organ during a liver transplantation and that shall be the topic of future communication from our research group. 


\section{Liver metabolic level obtained by optical fluorescence}

While a considerable amount of knowledge is available concerning the biochemical events in the liver regeneration, not everything related to the complex cellular kinetics of the compensatory growth is known. Normally, conventional analytical techniques are limited to the resolution attained in following enzymes and other biochemical parameters at the latest stage of the organ regeneration. Important parameters like cell population, mitoses index, proliferation rates among others cell activities are important to understand the liver regeneration. Here, we present a proposal of a new alternative to follow the metabolic activity through the overall fluorescence followed by laser excitation. We have applied and compared our results with mass gain, histological and biochemical investigation of liver because that is a topic of current interest within our research center. Nevertheless, the technique here employed can be used for the investigation of the transient metabolic activity of any biological tissue. First is important to point out that during our procedure to obtain the fluorescence spectra (Figure 7), we test in each data taken, a small fraction of the tissue. Knowing the depth of light penetration and the sensitivity of our device, we estimate that less than $1 \mathrm{~mm}^{3}$ is actually sampled during each examination. This volume is normally the one in contact with the optical fiber delivering the light and collecting the fluorescence.

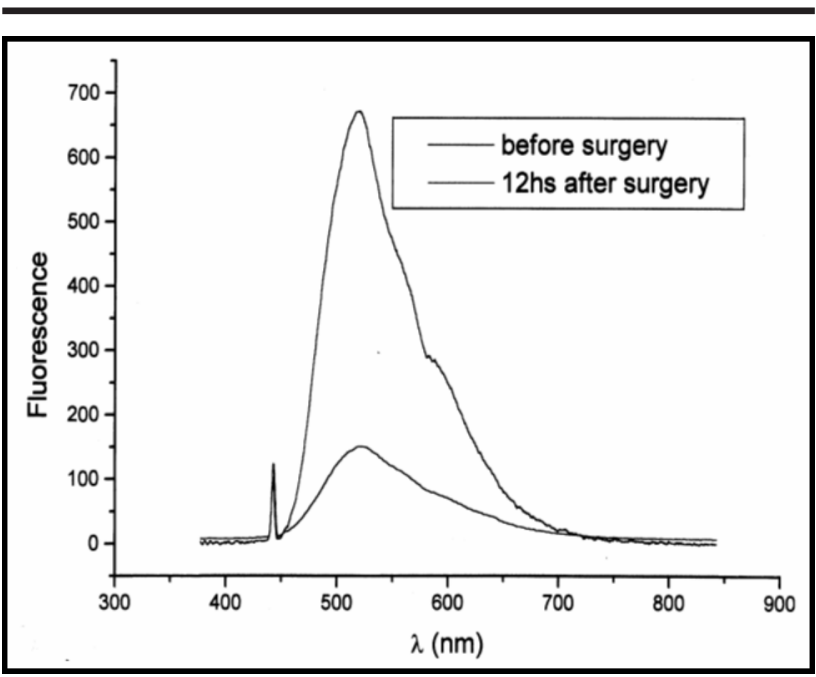

FIGURE 7 - Compare the fluorescence spectrum from a single animal excited at $442 \mathrm{~nm}$, before and $12 \mathrm{~h}$ after partial hepatectomy.

The overall spectra shows a first peak around the excitation wavelength, which we refer to as elastic scattering peak, followed by a broad peak at longer wavelength which corresponds to the fluorescence peak. On figure 8 we compare the fluorescence spectrum from a single animal excited at $442 \mathrm{~nm}$, before and $12 \mathrm{~h}$ after partial hepatectomy. Clearly, the intensity of the fluorescence of the normal tissue is much higher than the hepatectomized one when compared relatively to the elastic peak. After this normalization procedure, we notice that for either excitation source, the overall intensity of fluorescence drops dramatically indicating possible lowering of metabolic activity. Following the information available in the literature, this green type fluorescence is associated with the level of reduced NADH. Due to this difference, we can define a figure of merit (FM) to differentiate the cell activity before and after procedure. Considering the fluorescence spectrum we can define the mathematical operation equivalente to the ones already described in this paper. The figure 8 presents the evolution a figure of merit extracted from the spectra with the waiting time after partial hepatectomy. The first data point at $0 \mathrm{~h}$ coincides with the normal liver level of fluorescence. The period of $12 \mathrm{~h}$ after hepatectomy the fluorescence level drops by about a factor of 4 . This suddenly decrease of cell activity is also reported by other authors. This first period is associated with the occurrence of hypertrophy of the cytoplasm bodies as well as the nuclei. Around $12 \mathrm{~h}$ after, histological exams revels the beginning of mitoses. That is also the point where DNA synthesis is first labeled. This moment is followed by a rapid rise in the percentage of labeling index for mitoses. The binuclear cells are responsible for a great deal of cell activity. This activity is reveled in the fluorescence spectrum by the sudden increase in the value of the FM. The cell seems to increase the rate of activity around $36 \mathrm{~h}$, remaining at high level until about 50 $\mathrm{h}$, where a first decrease in the rate is noted. This period with an inflection on the rate and increase in the cell activity seems to correspond to the high level of cell division. In the literature, a second wave of cell activity towards DNA labeling and mitoses are observed after around $60 \mathrm{~h}$. That could well be the interval observed between 50 and $100 \mathrm{~h}$, where the rate of cell activity is about the some as for the period after $12 \mathrm{~h}$. Following this period, there seems to be another wave of intense activity, probably associated with the intense process of cell division and proliferation, this part is not reported by other authors because they may not have enough resolution to see it.

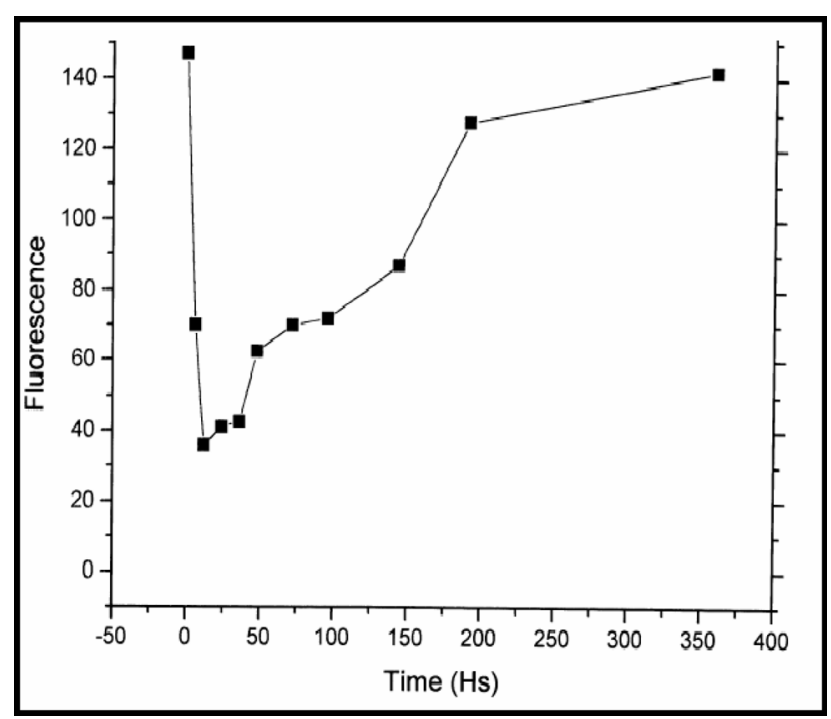

FIGURE 8 - The evolution a figure of merit extracted from the spectra, with the waiting time after partial hepatectomy. 
We believe that this second period going from $100 \mathrm{~h}$ to $200 \mathrm{~h}$ after hepatectomy is associated with the fact that the liver is not gaining more mass, however, is not yet fully able to account for the whole body activity. The period going up to $100 \mathrm{~h}$ is called by us the restoration period. Here, the main mass of the liver is attained due to parenchyma cells around the most outer zone relative to the portal triad and central vein, the inner zone cells comes slowly after the outer zone one. After this period the liver does not gain significantly more mass, but it is not yet fully recovered. It needs a little more mass to account for the full animal body mass of fluid. That is the reason for the second increase of rate in the cell activity. The period going from 100 to $200 \mathrm{~h}$ is called by us the period of equilibrium. It is a period of intense cell activity detected by fluorescence but not by regular histology. It is possible that at this second stage the most outer zone cells are the ones with intense activity, not noted by regular histological examination that normally takes into account a considerable amount of liver but does not focus on the surface of parenchymal cells. Fluorescence, however, observes these cells more than the inner ones and can detect the intense activity going on. It is important to note that during the restoration period the liver gets back most of the original mass. The period that goes on after $200 \mathrm{~h}$ is called the stabilization period. The fluorescence shows that at this last level the hepatocyte activities are still not as intense as the normal liver. Conventional techniques of labeling and biochemical analyses did not observe such a period and assume that in about 150 to $200 \mathrm{~h}$ the liver is fully recovered and all metabolic levels are similar to the normal liver. That does not seem to be the case looking with a more precise technique, in this case the overall fluorescence. The liver can be considered to have returned to normal metabolic activity about $350 \mathrm{~h}$ after the hepatectomy procedure. This work has suggested the capability of using fluorescence technique as a tool for evaluation of metabolic conditions on liver. Further investigation on this line is presently under progress.

\section{Overall conclusions}

The modern applications of light as a tool for diagnosis and treatment of liver dysfunctions seems very realistic and not far from the day to day clinical practice. The spectrum signature of each type of lesion must be obtained to allow the determination of the spectrum information relevant and inherent to the discrimination of such pathology. This can enable the detection of a lesion through an optical analysis, reducing the need of an invasive procedure or this can present important information to establish a diagnosis of a doubtful lesion. As in any other diagnostic procedures, the fluorescence detection must also be validated; its sensitivity and specificity tested, and that is our present concern. With respect to PDT treatment for cancer of the liver, it is turning a reality based on scientific results.

\section{References}

1. Vacca RA, Marra E, Quagliariello E, Greco M. Activation of mitochondrial DNA replication by He-Ne irradiation. Biochem Biophys Res Commun. 1993;95:704:9.

2. Belkin M, Schwartz M. Evidence for the existence of lowenergy laser bioeffects on the nervous system. Neurosurg Ver. 1994;17:7-17.

3. Anders JJ, Borke RC, Woolery SK, Van de Merwe WP. Low power laser irradiation alters the rate of regeneration of the rat facial nerve. Lasers Surg Med. 1993;13:72-82.

4. Karu TI, Pyatibrat LV, Kalendo GS, Serebriakov NG. Changes in the amount of ATP in Hela cells under the action of He-Ne laser radiation. Bull Eksp Biol Med. 1993;115:617-8

5. Castro e Silva O Jr., Zucoloto S, Menegazzo LAG, Granato RG, Marcassa LG, Bagnato VS. Laser Enhancement in hepatic regeneration for partially hepatectomized rats. Lasers Surg Med. 2001; 29:73-7.

6. Allison RR, Mota HC, Sibata CH. Clinical PD/PDT in north america: an historical review. Photodiag Photodyn Ther. 2004;1:263-77.

7. Wang HW, Putt ME, Emanuelle MJ, Glatstein E, Yodh AG, Busch TM. Treatment induced changes in tumor oxygenation predict photodynamic therapy outcome. Cancer Res. 2004; 64:7553-61.

8. Melo CA, Kurachi C, Clovis G, Sibata CH, Castro e Silva O Jr., Bagnato VS. Pharmacokinetics of photogem using fluorescence monitoring in wistar rats. Photochem Photobiol. 2004; 73: 183-8.

9. Alfano R, Tata D, Cordero J, Tomashefsky P, Longo F, Alfano M. Laser induced fluorescence spectroscopy from native cancerous and normal tissue. IEEE J. Quantum Electron. 1984; 20: 1507-11.

10. Gillenwater A, Jacob R, Kortum RR. Fluorescence spectroscopy: A technique with potential to improve the early detection of aerodigestive tract neoplasia. Head Neck. 1998; 20:556-62.

11. Broglia M. Blue-green laser induced fluorescence from intact leaves. Appl. Opt. 1993; 32: 334-8.

12. Dhingra JK; Perrault Jr DF;. McMillan K;. Rebeiz E. E;. Kabani S;. Manoharan R;. Itzkan I;. Feld M. S;. Shapshay S. M. Early diagnosis of upper aerodigestive tract cancer by autofluorescence. Arch. Otolaryngol. Head Neck Surg. 1996; 122: 1181-6.

13. Wagniéres GA, W. M. Star, and B. C. Wilson. In vivo fluorescence spectroscopy and imaging for oncological applications. Photochem. Photobiol. 1998; 68, 603-32

14. Vari SC Papazolou TG, der Veen MJ, Fishbein MC, Young FJD, Chandra M, Papaioannou T, Beeder C, Wei-Qiang Shi, Grundfest WS Intraoperative metastases detection by laser induced fluorescence spectroscopy, Proc. SPIE 1426, 1991:111-20;

15. Solt D. B and. Farber F. F. New principle for the analysis of chemical carcinogenesis, Nature 263, 1976;701-3

16. Marcassa JC, Ferreira J, Zucoloto S, Castro e Silva O, Marcassa LG. Bagnato VS. Detection of hepatocarcinoma 
in rats by integration of the fluorescence spectrum: experimental model. Lãs. Phys., 2006; 16. 827-32;

17. Zonios G CR, Crawford JM, Fitzmaurice M, Manoharan R, Van Dam J, Feld MS. Spectral pathologya [Advances in optical biopsy and optical mammographya: Part III. Flourescence and spectroscopy]. Ann NY Acad Sci, 1998; 838: 108-15;

18. Vo-Dinh T PM, Overholt BF. Laser-induced fluorescence for esophageal cancer and dysplasia diagnosis. Ann NY Acad Sci, 1998; 838: 116-22;

19. Anidjar MCO, Avrillier S, Ettori D, Teillac P, Le Duc A. The role of laser-induced autofluorescence spectroscopy in bladder tumor detection: dependence on the excitation wavelength. Ann NY Acad Sci, 1998; 838: 130-42;

20. Thomsen S TD. Physiological and pathological factors of human breast disease that can influence optical diagnosis [Advances in optical biopsy and optical mammographya: Part IV. Pathology and correlation studies]. Ann NY Acad Sci, 1998; 838: 171-93;

21. Larionov PMMA, Mandrik MM, Maslov NA, Orishich AM. Changes in laser-induced flourescence spectrum of myocardium tissue with decrease in it's viability. J. Appl. Spectroscopy 2003; 70: 38-42.

\section{Correspondence:}

Orlando de Castro e Silva Jr.

Rua Campos Salles, 809/9 ${ }^{\circ}$ andar

14015-110 Ribeirão Preto - SP Brazil

orlando@.fmrp.usp.br
Conflict of interest: none Financial source: CNPq and FAPESP

\section{How to cite this article}

Bagnato VS, Kurachi C, Ferreira J, Sankarankutty AK, Zucoloto S, Castro e Silva O. New photonic technologies for the treatment and diagnosis of hepatic diseases: An overview of the experimental work performed in collaboration, between Physics Institute of São Carlos and Ribeirão Preto Faculty of Medicine of University of São Paulo. Acta Cir Bras. [serial on the Internet] 2006;21 Suppl 1. Available from URL: http://www.scielo.br/acb 